\title{
Operando monitoring transition dynamics of responsive polymer using optofluidic microcavities
}

\author{
Da-Quan Yang ${ }^{1}$, Jin-hui Chen ${ }^{2}$, Qi-Tao $\mathrm{Cao}^{3}$, Bing Duan ${ }^{1}$, Hao-Jing Chen $\mathbb{1}^{3}$, Xiao-Chong Yu ${ }^{4}$ and Yun-Feng Xiao $\mathbb{1}^{3,5,6}$
}

\begin{abstract}
Optical microcavities have become an attractive platform for precision measurement with merits of ultrahigh sensitivity, miniature footprint and fast response. Despite the achievements of ultrasensitive detection, optical microcavities still face significant challenges in the measurement of biochemical and physical processes with complex dynamics, especially when multiple effects are present. Here we demonstrate operando monitoring of the transition dynamics of a phase-change material via a self-referencing optofluidic microcavity. We use a pair of cavity modes to precisely decouple the refractive index and temperature information of the analyte during the phase-transition process. Through real-time measurements, we reveal the detailed hysteresis behaviors of refractive index during the irreversible phase transitions between hydrophilic and hydrophobic states. We further extract the phase-transition threshold by analyzing the steady-state refractive index change at various power levels. Our technology could be further extended to other materials and provide great opportunities for exploring on-demand dynamic biochemical processes.
\end{abstract}

\section{Introduction}

Micro-/nano-photonic devices have provided increasing opportunities for optical sensing and detection in the past decades, with intriguing features of miniature size, noninvasiveness and fast response, etc. ${ }^{1-9}$. In particular, high$Q$ microcavities manifest themselves by virtue of the strongly enhanced light-matter interaction, and have witnessed tremendous progress of ultrasensitive optical sensing ${ }^{10-17}$. For example, the detection limit of the microcavity sensors has reached levels of single molecules or single ions by employing mode-locking, optical spring, or plasmonic enhancement methods ${ }^{18-21}$. Despite their achievements of ultrahigh sensitivities, the conventional schemes of microcavity sensing, for example, by monitoring the shift of a single resonance mode ${ }^{12,22}$, usually

\footnotetext{
Correspondence: Yun-Feng Xiao (yfxiao@pku.edu.cn)

${ }^{1}$ School of Information and Communication Engineering, State Key Laboratory of Information Photonics and Optical Communications, Beijing University of Posts and Telecommunications, Beijing 100876, China

${ }^{2}$ Institute of Electromagnetics and Acoustics, Xiamen University, Xiamen 361005, China

Full list of author information is available at the end of the article

These authors contributed equally: Da-Quan Yang, Jin-hui Chen, Qi-Tao Cao
}

cannot identify mixed effects. Thus, most current optical microsensors are restricted to monitoring a single chemical/physical measurand at a time $\mathrm{e}^{23-25}$. To measure more complicated processes involving multi-physical quantities, new detection technology with straightforward signal deconvolution capability is required urgently.

In this work, we experimentally monitor the transition dynamics of poly(N-isopropylacrylamide) (PNIPA), a typical phase-change material, by using a high- $Q$ optofluidic microcavity, in which the integrated microfluidic channel allows efficient coupling between the resonant optical field and the PNIPA molecules for operando detection. By developing a self-referencing strategy with dual-modes of the microcavity, the changes of temperature and refractive index of PNIPA during the phase transition are individually extracted from the cavityresonance spectra. In real-time measurements, the refractive index of PNIPA demonstrating a hysteresis phenomenon in the cycles between hydrophilic and hydrophobic states is observed for the first time. In addition, under thermal-equilibrium conditions, it is found that the refractive index of PNIPA exhibits a

\section{(c) The Author(s) 2021}

(c) (i) Open Access This article is licensed under a Creative Commons Attribution 4.0 International License, which permits use, sharing, adaptation, distribution and reproduction cc) in any medium or format, as long as you give appropriate credit to the original author(s) and the source, provide a link to the Creative Commons license, and indicate if changes were made. The images or other third party material in this article are included in the article's Creative Commons license, unless indicated otherwise in a credit line to the material. If material is not included in the article's Creative Commons license and your intended use is not permitted by statutory regulation or exceeds the permitted use, you will need to obtain permission directly from the copyright holder. To view a copy of this license, visit http://creativecommons.org/licenses/by/4.0/. 
classical Boltzmann distribution depending on the heating power and manifests the threshold of the phase transition. This strategy combines microcavity photonics with microfluidics and phase change materials, in which not only the basic properties of phase change materials are well characterized with dual-mode, self-referencing spectra, but also the functional photonic devices, such as optical switches ${ }^{26}$ and optical memories ${ }^{27}$, can be constructed.

\section{Results}

PNIPA is a prototype thermo-responsive polymer and has drawn extensive attention in the applications of medicine, bioelectronics, optics, and robotics ${ }^{28-32}$. Below the lower critical solution temperature (LCST), the hydrophilic amide group of PNIPA is connected with water via hydrogen bond and exists as a fully hydrated random coil. As the temperature exceeds the LCST, PNIPA experiences a deswelling phase transition and transforms from the hydrophilic state to the hydrophobic state $^{33,34}$ (Fig. 1a inset), mainly accompanied by a refractive index variation and heat transfer. In our experiment, 25\% PNIPA solution (refractive index 1.33) is prepared by polymerization of monomers (see Methods) and then injected into a silica hollow bubble microcavity (diameter $\sim 80 \mu \mathrm{m}$ ) serving as a microfluidic channel for in operando monitoring the phase transition of PNIPA, after which the microbubble cavity is sealed using ultraviolet glue.

As shown in Fig. 1a, bubble microcavities support whispering-gallery modes (WGMs) via total internal reflection ${ }^{35-37}$. A tapered microfiber is used to evanescently couple the probe light at $780 \mathrm{~nm}$ (linewidth $<200 \mathrm{kHz}$, power $<0.11 \mathrm{~mW}$ ) into the microcavity, and the transmission spectra of the resonant modes are measured, confirming $Q$ factors over $10^{6}$ (see details in Supplementary Information). An external continuous infrared laser at $1550 \mathrm{~nm}$ is employed to locally heat the PNIPA solution and induce phase transition in a short time with a heating rate of $2-8{ }^{\circ} \mathrm{C} \mathrm{s}^{-1}$ (Fig. S2 in Supplementary Information), resulted from the light absorption of the solution with an absorptivity of $7.667 \mathrm{~cm}^{-138}$. Note that the pump light and probe light do not interfere with each other, because they are from two independent diode lasers, with different wavelengths and incoherent phases. The experiments are conducted in a cleanroom with the temperature of $\sim 22^{\circ} \mathrm{C}$ and the humidity of $\sim 40 \%$. As shown in Fig. 1b, during the cyclic phase transition process between the hydrophilic and hydrophobic states, resonance mode of the bubble microcavity experiences a continuous shift due to the change of both refractive index and temperature of PNIPA. Since the cavity resonances are encoded with the structural transition information of PNIPA, the WGM spectra exhibit nonmonotonic red-shift (blue-shift) in the heating (cooling) process. To verify the repeatability of our experimental setup, we carry out the experiment for over ten times with different microcavity devices at the same measurement condition, and the measurement results of each experiment are similar.

Here, the wavelength shift of the WGM sensor is simultaneously influenced by the thermo-optic effect and phase transition of PNIPA. Thus, it is a non-trivial task to resolve the contributions from each effect by solely tracing the spectrum of one WGM. To overcome this challenge, we develop a self-referencing sensing strategy to separately extract the two different contributions by simultaneously analyzing the shifts of a reference mode (RM) and a sensing mode (SM) which can be experimentally identified through mode broadening mechanism $^{39}$. By further analyzing the field distribution in COMSOL simulation, it is calculated that over $99.94 \%$ optical field of the reference mode are confined in the solid wall of the silica bubble (shown in Fig. 2a, top), so
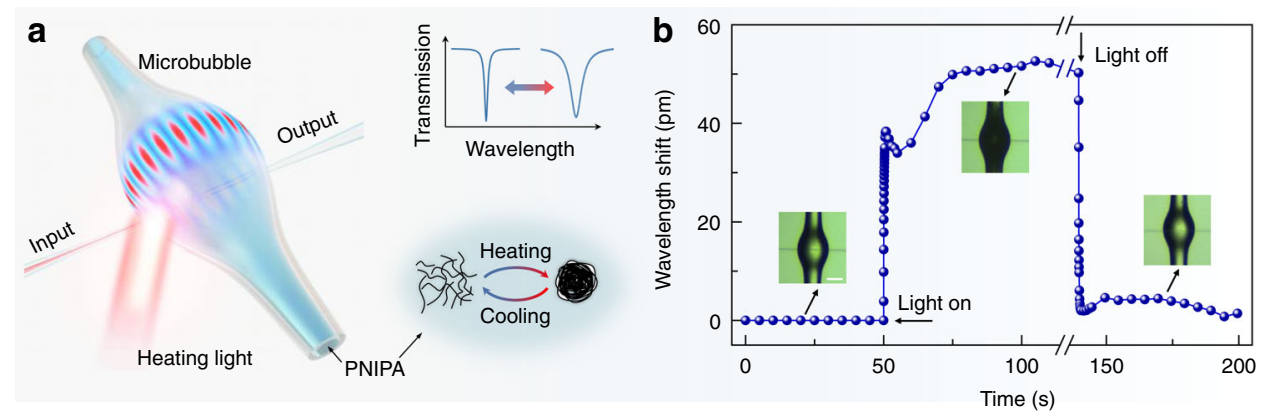

Fig. 1 Optofluidic microcavity for PNIPA sensing. a Schematic of microbubble sensors for in operando monitoring of phase transition dynamics. Inset: the variation of WGM spectra correlated to the mesoscopic structures of PNIPA, where the cyclic phase transition changing between hydrophilic state and hydrophobic state. $\mathbf{b}$ Time-resolved wavelength shift of a typical WGM sensor during one cycle of heating-light on and off. Inset: Optical microscope images of PNIPA-microbubble at different stages, in which the bright (dark) image indicates hydrophilic (hydrophobic) state. The scale bar is $40 \mu \mathrm{m}$ 

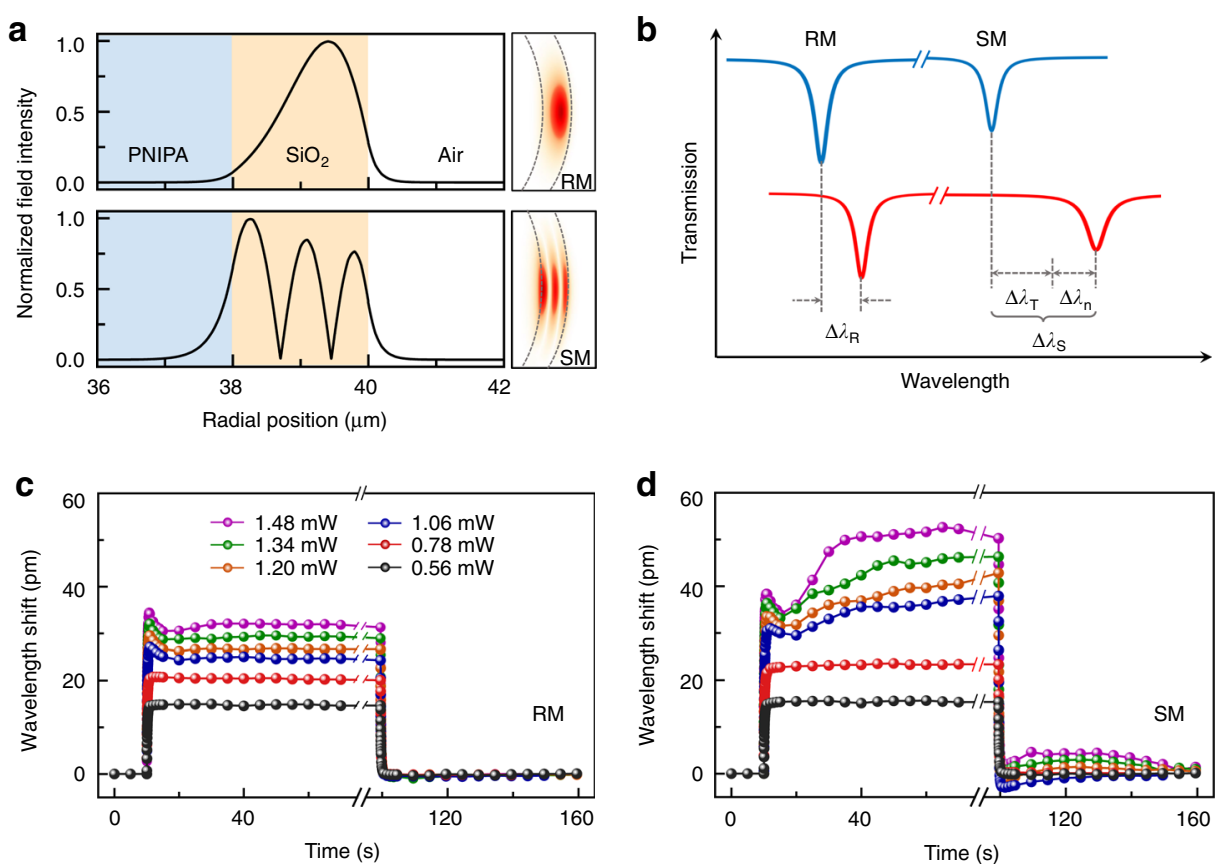

Fig. 2 Concept of self-referencing WGM sensors. a Electrical field (|E|) distribution of reference mode (RM) and sensing mode (SM) by finiteelement-method simulation. b Schematic of self-referencing strategy with dual-WGMs sensing method, where $\Delta \lambda_{T}=\left(\beta_{2} / \beta_{1}\right) \cdot \Delta \lambda_{R}$. c , d Real-time wavelength shifts of reference mode and sensing mode at different heating power and natural cooling $\left(@ 22^{\circ} \mathrm{C}\right)$, respectively

that the resonance shift mainly arises from the absorption of the heating light and the subsequent refractive index change by the thermo-optic effect denoted as $\Delta \lambda_{R}$ (Fig. $2 b)$. In contrast, there is a considerable part $(\sim 6.0 \%$, two-orders of magnitude larger than that of the reference mode) of evanescent field penetrating into the PNIPA for the sensing mode (Fig. 2a, bottom), and the resonance shift $\Delta \lambda_{S}$ is caused by the thermo-optic effect $\Delta \lambda_{T}$ and intrinsic refractive index change $\Delta \lambda_{n}$ of PNIPA (Fig. 2b). Here the thermo-optic effect induced mode shifts of sensing and reference mode are usually different due to the distinct field distributions, but hold a constant ratio $\beta_{2} / \beta_{1}$, which is derived from the experimental measurement. In this way, the mode shift by the intrinsic refractive index change of PNIPA during the phase transition is derived (see details in Methods):

$$
\Delta \mathcal{\lambda}_{n}=\Delta \mathcal{U}_{S}-\Delta \mathrm{\lambda}_{T}=\Delta \mathrm{U}_{S}-\frac{\beta_{2}}{\beta_{1}} \Delta \mathrm{\lambda}_{R}
$$

Therefore, the temperature and refractive index of PNIPA during the phase transition can be deciphered by the dual-WGMs sensor.

Experimentally, we monitor the spectral shifts of both the reference mode and sensing mode in real time upon optical heating (response time $\sim 0.4 \mathrm{~s}$ ) and ambient cooling operations (response time $\sim 1.0 \mathrm{~s}$ ), as shown in Fig. 2c, d. Under the weak heating power (red and black curves), both the reference mode and sensing mode experience red shifts rapidly after the heating light is on within the time scale $\sim 0.4 \mathrm{~s}$, mainly arising from the thermo-optic effect. As seen, the sensing mode shifts a bit larger than reference mode with a ratio of $\beta_{2} / \beta_{1} \sim 1.03$ because of the different field distributions. Then, the resonances of the two modes remain at the constant wavelengths, indicating that the temperature reaches an equilibrium state.

With the further increase of the heating power, small blue-shifts emerge after the rapid red shift for both reference and sensing modes, since the deswelling transition absorbs the heat from the environment and results in the temperature decrease ${ }^{40}$. Then, during the deswelling transition process from the hydrophilic state to hydrophobic state, the reference mode reaches thermal equilibrium and stops shifting as time goes on, while, in contrast, the sensing mode keeps shifting towards longwavelength. The aforementioned phenomena of the PNIPA solution under high heat power are absent in the case of microbubble filled with deionized water (Fig. S3c in Supplementary Information), indicating the exclusive effects of this phase transition dynamics. When the heating light is off, both the reference and sensing mode experience the fast blue-shift within the time scale $\sim 1.0 \mathrm{~s}$. Afterwards, the reference mode quickly reaches a stable state, while sensing mode exhibits slow red-shift and then gets its stable state which is probably due to the liquid convection inside the microbubble ${ }^{41}$. The physical 
a

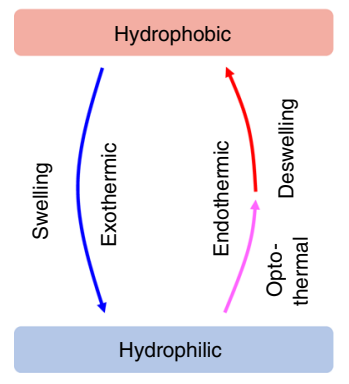

b

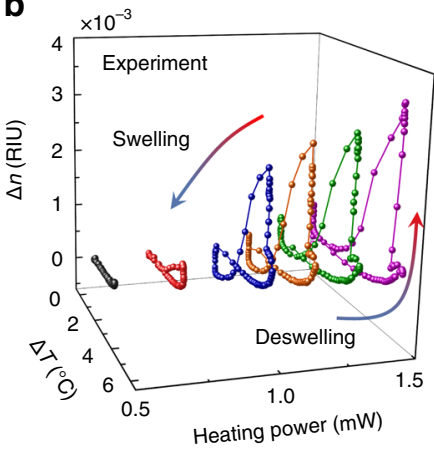

C

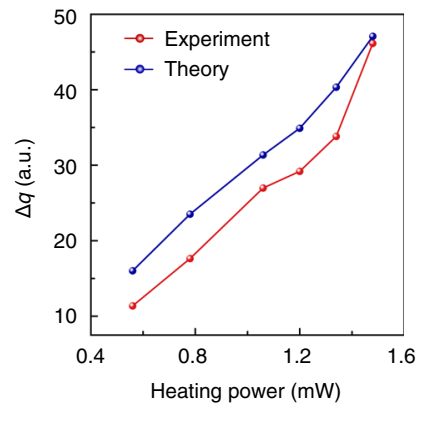

Fig. 3 Probing the phase transition dynamics of PNIPA. a Diagram of phase transition process of PNIPA. The opto-thermal effect by infrared light increases the temperature of PNIPA solution, then it activates the de-swelling transition. The hydrophobic to hydrophilic transition is a direct swelling process by ambient cooling. $\mathbf{b}$ Dependence of the refractive index change on the temperature of PNIPA solution at different heating power in the experiment. c Dissipated heat $(\Delta q)$ in a transition cycle vs. the heating power by the experimental measurement (red curve) and theoretical calculation (blue curve)

processes of PNIPA phase transition triggered by illuminated infrared light are illustrated in Fig. 3a.

Furthermore, we separately extract the refractive index and temperature changes and reveal a clear hysteresis phenomenon during the PNIPA deswelling-swelling transition cycle. At each heating power, the PNIPA solution absorbs the light, and both the temperature and refractive index of the PNIPA solution change simultaneously, of which the dynamical evolution process is tracked and illustrated in the parametric space spanned by temperature and refractive index, as shown in Fig. 3b. In the heating-cooling cycle with the weak infrared light (for example, the black curve), the temperature increases and decreases reversibly, while the intrinsic refractive index keeps nearly unchanged, demonstrating that the phase transition does not happen at this stage. When the heating power is high enough (for example, the blue curve in Fig. 3b), the hydrophilic PNIPA absorbs energy under the heating operation, and the temperature rises until the emergence of the deswelling process. Subsequently, the temperature decreases by less than $0.5^{\circ} \mathrm{C}$, and meanwhile the refractive index of the PNIPA solution begins to increase, featuring the start of the deswelling transition. In the following process, the temperature remains constant, while the intrinsic refractive index increases monotonously, which is contrary to the initial process dominated by the thermo-optic effect. This phenomenon denotes the isothermal characteristics of the phase transition from the hydrophilic state to the hydrophobic state.

When the heating light is turned off, distinct from the deswelling process, the PNIPA solution experiences a swelling transition with the slow decline of intrinsic refractive index change relating to the temperature. Notably, this kind of irreversible transition forms a pronounced hysteresis loop of refractive index in a closed cycle, which is the typical behavior in phase transitions of responsive polymers ${ }^{33,42,43}$. Although the previously reported hysteresis was obtained from the measurement of hydrodynamic radius ${ }^{34}$, heat capacity ${ }^{44}$, and infrared absorption fingerprint ${ }^{43}$, rather than the refractive index, the hysteresis phenomena by different approaches are attributed to the similar mechanism, i.e., the formation of extra intra- and inter-chain hydrogen bonds as the PNIPA coils collapsed to globules ${ }^{33}$. Besides, it is also noticed that an additional hysteresis loop appears at the end of the swelling transition, probably due to the solution convection inside the microbubble. We note that this is the first report of the hysteresis effect in phase change materials by using the microcavity sensor, to the best of our knowledge. According to the theoretical analysis based on FloryRehner theory (see Methods and Fig. S5 in Supplementary Information), it is found that the encircled area of major hysteresis loop in the transition cycles corresponds to the dissipated heat in the cycle of structural transition, with respect to the irreversible fracture and formation of hydrogen bonds ${ }^{34}$. Besides, it is also found that the dissipated heat is positively associated with the heating power (shown in Fig. 3c), because the heat capacity of PNIPA is nearly proportional to the polymer volume fraction which is correlated to the heating power ${ }^{44}$.

Besides the dynamic reaction of the phase transition, we also interrogate the steady-state response of PNIPA under the heating operation with different powers (Fig. 4). Experimentally, at each heating power, the eventual wavelength shifts of both the reference mode and sensing mode are recorded after about $20 \mathrm{~s}$ which is enough for thermal equilibrium, as shown in the inset of Fig. 4b. Note that here the required time for thermal equilibrium is different from that in the measurements in Fig. $2 \mathrm{c}-\mathrm{d}$ (for example, over $50 \mathrm{~s}$ at $1.06 \mathrm{~mW}$ ), because each measurement at a certain power in Fig. 4a begins with a bias- 

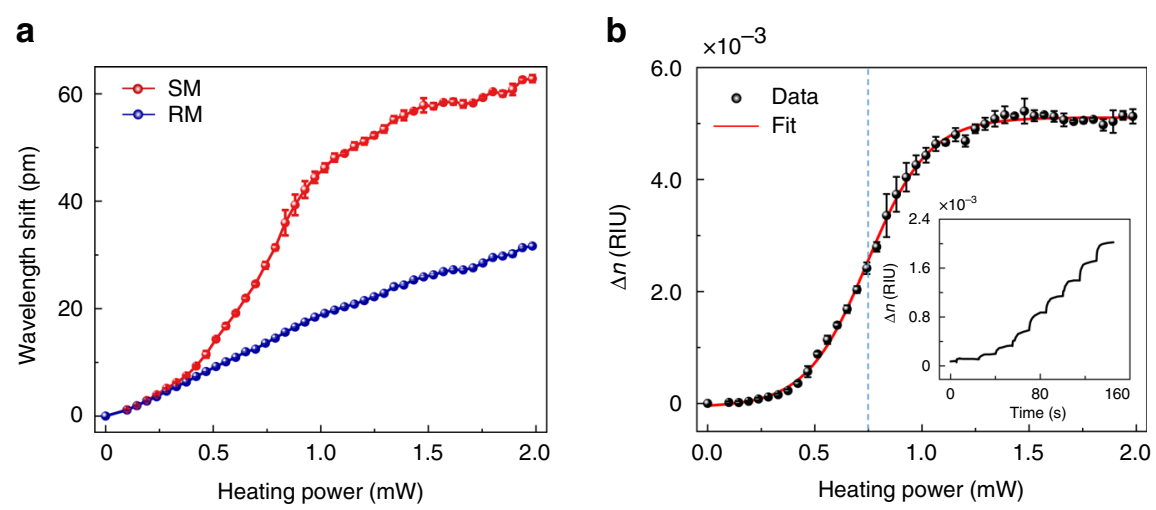

Fig. 4 Steady-state phase transition dynamics. a Dependence of the wavelength shifts of reference mode and sensing mode on the heating light power. $\mathbf{b}$ Extracted refractive index change $(\Delta n)$ of PNIPA solution on the heating light power. Inset: Extracted real-time refractive index change as the heating light power is stepwise increased

heating power. Under the weak heating light lower than $0.5 \mathrm{~mW}$, both the sensing mode and reference mode experience linear wavelength shifts approximately, with a slope ratio of $\beta_{2} / \beta_{1}$. As the heating power further rises, the reference mode still shifts linearly, whereas the sensing mode significantly deviates from linear shifts due to the occurrence of the deswelling phase transition. The information of the intrinsic refractive index is further extracted through de-convolving its thermo-optic response, as plotted in Fig. $4 \mathrm{~b}$. The dependence of the refractive index on the heating power follows the classical Boltzmann distribution ${ }^{45}$, where the threshold power of the transition is calculated as $0.75 \mathrm{~mW}$ (blue dashed line). It is also found that the saturation of the refractive index emerges gradually with the heating power higher than $1.5 \mathrm{~mW}$, indicating that all the PNIPA inside the microbubble transforms into the hydrophobic state.

\section{Discussion}

In summary, we have experimentally investigated the phase-transition dynamics of the responsive polymer PNIPA by using an ultrahigh- $Q$ microfluidic cavity. Benefitting from a self-referencing strategy employing dualWGMs, the information of the refractive index and temperature of PNIPA during the phase transition is successfully decoded from the transmission spectra of the microcavity. Consequently, a hysteresis phenomenon of the refractive index change is observed in the cycle of deswelling-swelling transition, of which the encircled area corresponds to the dissipated heat. Additionally, the steady-state evolution is analyzed, exhibiting a Boltzmann distribution with a transition threshold of $0.75 \mathrm{~mW}$.

Besides our present method by microcavities, some other techniques have also been developed for monitoring phase-transition dynamics of responsive polymers, including calorimetry ${ }^{46}$, rheology ${ }^{47}$, and light scattering ${ }^{48}$. Nevertheless, all the existing methods are hampered by low sensitivity and slow response, so that some slightly fast variations in phase transition process are challenging to be observed, for example, the weak endothermic process measured in the initial deswelling transition in Fig. $3 \mathrm{~b}$. On the contrast, the microcavity sensors proposed in this work not only possess the universal merits (e.g., miniature footprint and fast response) of micro-/nanophotonic devices, but also manifest a lot of unique shining points. For instance, the multimode property of the WGM microcavity enables the simultaneous monitoring of multi-physical features in a single platform, and the ultrahigh- $Q$ resonance allows trace measurements with a tiny amount of analyte. Besides, the compatibility with microfluidic systems can support small-volume sample handling for performing automated functions. We would also point out that this work is a fundamental proof-ofprinciple study, which can be readily extended to probe the phase transition of other phase-change materials, such as $\mathrm{GeSbTe}^{49-51}, \mathrm{SrTiO}_{3}{ }^{52}, \mathrm{VO}_{2}{ }^{53}$, and provide opportunities for exploring novel dynamic biochemical processes such as protein denaturation.

\section{Materials and methods \\ PNIPA linear polymer preparation}

The poly(N-isopropylacrylamide)-based polymer (PNIPA) was prepared as follows: Firstly, $10 \mathrm{~g}$ of $\mathrm{N}$ isopropylacrylamide (NIPA) monomer was dissolved in $50 \mathrm{~mL}$ benzene with $0.16 \mathrm{~g}$ AIBN added as an initiator. The polymerization was carried out in water bath at $65{ }^{\circ} \mathrm{C}$ for $10 \mathrm{~h}$ under $\mathrm{N}_{2}$ protection. After polymerization, the benzene was removed by evaporation. The resulting crude polymer was further dried and dissolved in a small amount of acetone and then was put into $n$ hexane drop by drop. After the filtration, it was dried under a vacuum environment at $30^{\circ} \mathrm{C}$ for $48 \mathrm{~h}$ and the white solid of PNIPA was obtained. Finally, the PNIPA solution was successfully prepared by dissolving the 
solid in deionized water. The average molecular mass of $1.65 \times 10^{4} \mathrm{~g} \mathrm{~mol}^{-1}$ was measured by Agilent 1100 series gel chromatograph.

\section{Fabrication of microbubble cavity}

The microbubble cavity is fabricated by the following steps. First, the fused-silica capillary with an outer diameter of $140 \mu \mathrm{m}$ and inner diameter of $100 \mu \mathrm{m}$ was tapered to an outer diameter of $30 \mu \mathrm{m}$ using a heat-andpull method. Next, the counter-propagating $\mathrm{CO}_{2}$ laser beams were focused onto the internally pressurized capillary, where the focused region expanded and the wall became thinner. Finally, a microbubble cavity with a diameter of $\sim 80 \mu \mathrm{m}$ and a wall thickness of $2.0-2.5 \mu \mathrm{m}$ was formed by controlling the heating parameters.

\section{Theoretical models of self-referencing WGM sensor}

By using a self-reference sensing strategy, we can eliminate thermal response and then obtain the intrinsic refractive index change purely induced by PNIPA structural transition. The PNIPA refractive index change contributing to the reference mode is two orders of magnitude smaller than that of the sensing mode, so it is ignored in the calculation. The wavelength shifts of RM and SM are expressed as:

$$
\begin{aligned}
& \Delta \mathcal{U}_{\mathrm{RM}}=\lambda_{1} \alpha_{1} \Delta T / n_{\text {eff }, 1}=\beta_{1} \Delta T \\
& \Delta \lambda_{\mathrm{SM}}=\lambda_{2} \alpha_{2} \Delta T / n_{\text {eff }, 2}+\gamma \Delta n=\beta_{2} \Delta T+\gamma \Delta n
\end{aligned}
$$

where $\Delta \lambda$ stands for wavelength shift, $\lambda_{i}$ is the WGM resonance wavelength $\sim 779.50 \mathrm{~nm}, \Delta T$ denotes the temperature variation of microbubble resonator, $\alpha_{1}$ is the effective themo-optic coefficients (TOC) of silica, $\beta_{1}=5.92 \mathrm{pm}^{\circ} \mathrm{C}^{-1}$ is the temperature sensitivity of the reference mode, $\alpha_{2}$ represents the weighted TOC of silica and PNIPA solution, $n_{\text {eff } i}$ is effective refractive index of WGM, $\gamma$ describes the refractive index change solely induced by structural transition. According to Eqs. (1) and (2), the $\Delta n$ induced by the phase transition of PNIPA is derived:

$$
\gamma \Delta n=\Delta \lambda_{\mathrm{SM}}-\frac{\beta_{2}}{\beta_{1}} \Delta \lambda_{\mathrm{RM}}
$$

where $\gamma=6151.14 \mathrm{pm} \mathrm{RIU}^{-1}, \beta_{2} / \beta_{1}=1.03$. Here, $\gamma$ is determined by the field distribution of sensing mode employing the finite element method in COMSOL. In the simulation, the light wavelength is set as $780 \mathrm{~nm}$, the diameter and wall thickness of the microbubble cavity are $80 \mu \mathrm{m}$ and $2.0 \mu \mathrm{m}$, respectively. The ratio of $\beta_{2} / \beta_{1}$ is directly extracted from experiments.

\section{Theoretical models of phase transition dynamics}

The phase-transition dynamics of PNIPA is modeled based on Flory-Rehner theory, which describes the transition kinetics of PNIPA polymer by balancing the elastic and mixing contributions to the osmotic pressure within polymers ${ }^{54}$. The Flory parameter $X$, describes the change of free energy when a solvent-solvent contact is replaced by a solvent-polymer contact, and is expressed as $^{55,56}$ :

$$
\begin{aligned}
& X=\frac{1}{2}-A\left(1-\frac{\theta}{T}\right) \\
& X=\frac{1}{\varphi^{2}}\left\{\eta\left[\frac{\varphi}{2 \varphi_{0}}-\left(\frac{\varphi}{\varphi_{0}}\right)^{1 / 3}\right]-\varphi-\ln (1-\varphi)\right\}
\end{aligned}
$$

where $A$ represents the entropy change, $\theta$ is the theta temperature depends on both entropic and enthalpic variations, $\eta$ is a dimensionless factor relating to the state of PNIPA $\left(\eta=5.26 \times 10^{-4}\right.$ in the heating process and $1.75 \times 10^{-4}$ in cooling process in this experiment), $\varphi$ is the polymer volume fraction and $\varphi_{0}$ is the polymer volume fraction at reference state $\left(\varphi_{0}=0.03\right.$ in the heating process and 0.01 in cooling process in this experiment), and the $\varphi$ can be calculated by Eqs. (4) and (5). In addition, the intrinsic refractive index change can be obtained by $\Delta n(T)=m \cdot \Delta \varphi(T)$, where $m=0.19^{57}$.

In addition, we extract the heat dissipation in the cycle of structural transition both experimentally and theoretically by introducing a parameter, i.e., specific heat capacity. Here, we assume that the specific heat capacity of PNIPA solution is the linear superposition of water and PNIPA polymer and the coefficients depend on the volume fraction of water and polymer, i.e.

$$
C(T)=(1-\varphi(T)) C_{\text {water }}+\varphi(T) C_{\text {polymer }}
$$

wherein $C_{\text {water }}=4.2 \mathrm{~J} \mathrm{~g}^{-1} \mathrm{~K}^{-1}$ and $C_{\text {polymer }}=4.93 \mathrm{~J} \mathrm{~g}^{-1}$ $\mathrm{K}^{-1}$ denotes the heat capacity of water and PNIPA polymer, respectively ${ }^{46}$. Thus, the heat dissipation under a close cycle of transition is calculated by $\Delta q=\oint C(T) \mathrm{d} T$.

\begin{abstract}
Acknowledgements
The authors acknowledge helpful discussions with Prof. Xiaoshi Qian, Prof. Cheng Wang, Dr. Wenjing Liu, Li-Kun Chen, and Aiqiang Wang. This work was supported by the National Key R\&D Program of China (No. 2018YFB2200401), the National Natural Science Foundation of China (Nos. 11825402, 11654003, 12041602, 11974058, and 62005231). D.-Q.Y. was also supported by Beijing Nova Program (Z201100006820125), Beijing Municipal Science \& Technology Commission (No. Z201100004020007), and Fundamental Research Funds for the Central Universities (20720200074). Q.-T.C. was also supported by the National Postdoctoral Program for Innovative Talents (No. BX20200014) and China Postdoctoral Science Foundation (No. 2020M680185). Some numerical simulations were performed at the High-performance Computing Platform of Peking University.
\end{abstract}

\section{Author details}

${ }^{1}$ School of Information and Communication Engineering, State Key Laboratory of Information Photonics and Optical Communications, Beijing University of Posts and Telecommunications, Beijing 100876, China. Institute of

Electromagnetics and Acoustics, Xiamen University, Xiamen 361005, China.

${ }^{3}$ State Key Laboratory for Mesoscopic Physics and Frontiers Science Center for 
Nano-optoelectronics, School of Physics, Peking University, Beijing 100871, China. ${ }^{4}$ Department of Physics and Applied Optics Beijing Area Major Laboratory, Beijing Normal University, Beijing 100875, China. ${ }^{5}$ Collaborative Innovation Center of Extreme Optics, Shanxi University, Taiyuan 030006, China. ${ }^{6}$ Peking University Yangtze Delta Institute of Optoelectronics, Nantong 226010, China

\section{Author contributions}

D.-Q. Yang, J.-h. Chen, and Q.-T. Cao contributed equally to this work. Y.-F. Xiao designed the experiment and supervised the project. All authors contributed to the experiment and discussion, analyzed the data, and wrote the manuscript.

\section{Conflict of interest}

The authors declare no competing interests.

Supplementary information The online version contains supplementary material available at https://doi.org/10.1038/s41377-021-00570-1.

Received: 20 April 2021 Revised: 15 May 2021 Accepted: 1 June 2021 Published online: 16 June 2021

\section{References}

1. Zhang, L., Tang, Y. \& Tong, L. Micro-/nanofiber optics: merging photonics and material science on nanoscale for advanced sensing technology. iscience $\mathbf{2 3}$ 100810 (2020).

2. Cao, Z. et al. Biochemical sensing in graphene-enhanced microfiber resonators with individual molecule sensitivity and selectivity. Light 8, 107 (2019).

3. Mauranyapin, N. P. et al. Evanescent single-molecule biosensing with quantum-limited precision. Nat. Photonics 11, 477-481 (2017).

4. Hoa, X. D., Kirk, A. G. \& Tabrizian, M. Towards integrated and sensitive surface plasmon resonance biosensors: a review of recent progress. Biosens. Bioelectron. 23, 151-160 (2007).

5. Stockman, M. I. Nanoplasmonic sensing and detection. Science $\mathbf{3 4 8}, \mathbf{2 8 7 - 2 8 8}$ (2015).

6. Frustaci, S. \& Vollmer, F. Whispering-gallery mode (WGM) sensors: review of established and WGM-based techniques to study protein conformational dynamics. Curr. Opin. Chem. Biol. 51, 66-73 (2019).

7. Zhou, J. J. et al. Single-particle spectroscopy for functional nanomaterials. Nature 579, 41-50 (2020).

8. Gopinath, A. et al. Engineering and mapping nanocavity emission via precision placement of DNA origami. Nature 535, 401-405 (2016).

9. Jiang, X. F. et al. Whispering-gallery sensors. Matter 3, 371-392 (2020).

10. Fan, X. D. et al. Sensitive optical biosensors for unlabeled targets: a review. Anal. Chim. Acta 620, 8-26 (2008).

11. Foreman, M. R., Swaim, J. D. \& Vollmer, F. Whispering gallery mode sensors. Adv. Opt. Photonics 7, 168-240 (2015).

12. Zhi, Y. Y. et al. Single nanoparticle detection using optical microcavities. Adv. Mater. 29, 1604920 (2017).

13. Tang, S. J. et al. Laser particles with omnidirectional emission for cell tracking. Light 10, 23 (2021).

14. Svela, A. O. et al. Coherent suppression of backscattering in optical microresonators. Light 9, 204 (2020).

15. Song, Q. H. Emerging opportunities for ultra-high $Q$ whispering gallery mode microcavities. Sci. China 62, 074231 (2019).

16. Liao, J. \& Yang, L. Optical whispering-gallery mode barcodes for highprecision and wide-range temperature measurements. Light 10, 32 (2021).

17. Liu, W. J. et al. Nonlinear sensing with whispering-gallery mode microcavities: from label-free detection to spectral fingerprinting. Nano Lett. 21, 1566-1575 (2021).

18. Toropov, N. et al. Review of biosensing with whispering-gallery mode lasers. Light 10, 42 (2021).

19. Baaske, M. D. \& Vollmer, F. Optical observation of single atomic ions interacting with plasmonic nanorods in aqueous solution. Nat. Photonics 10, 733-739 (2016).
20. Yu, W. Y. et al. Cavity optomechanical spring sensing of single molecules. Nat. Commun. 7, 12311 (2016).

21. Su, J., Goldberg, A. F. G. \& Stoltz, B. M. Label-free detection of single nanoparticles and biological molecules using microtoroid optical resonators. Light 5, e16001 (2016).

22. Vollmer, F. et al. Protein detection by optical shift of a resonant microcavity. Appl. Phys. Lett. 80, 4057-4059 (2002).

23. $\mathrm{Xu}, \mathrm{X}$. Y. et al. Wireless whispering-gallery-mode sensor for thermal sensing and aerial mapping. Light 7, 62 (2018).

24. Huang, Q. L. et al. Stretchable PEG-DA hydrogel-based whispering-gallerymode microlaser with humidity responsiveness. J. Lightwave Technol. 36, 819-824 (2018).

25. Li, B. B. et al. Quantum enhanced optomechanical magnetometry. Optica 5, 850-856 (2018)

26. Sui, G. R. et al. Microcavity-integrated graphene waveguide: a reconfigurable electro-optical attenuator and switch. Sci. Rep. 8, 12445 (2018).

27. Feldmann, J. et al. Parallel convolutional processing using an integrated photonic tensor core. Nature 589, 52-58 (2021).

28. Yuk, H., Lu, B. Y. \& Zhao, X. H. Hydrogel bioelectronics. Chem. Soc. Rev. 48 1642-1667 (2019)

29. Qiu, Y. \& Park, K. Environment-sensitive hydrogels for drug delivery. Adv. Drug Deliv. Rev. 53, 321-339 (2001).

30. Zhu, D. Y. et al. Repeatedly intrinsic self-healing of millimeter-scale wounds in polymer through rapid volume expansion aided host-guest interaction. ACS Appl. Mater. Interfaces 12, 22534-22542 (2020).

31. Wang, Y. et al. Chiral polypeptide thermogels induce controlled inflammatory response as potential immunoadjuvants. ACS Appl. Mater. Interfaces 11, 8725-8730 (2019).

32. Jochum, F. D. \& Theato, P. Temperature-and light-responsive smart polymer materials. Chem. Soc. Rev. 42, 7468-7483 (2013).

33. Cheng, H., Shen, L. \& Wu, C. LLS and FTIR studies on the hysteresis in association and dissociation of poly(N-isopropylacrylamide) chains in water. Macromolecules 39, 2325-2329 (2006).

34. Wu, C. \& Wang, X. H. Globule-to-coil transition of a single homopolymer chain in solution. Phys. Rev. Lett. 80, 4092-4094 (1998).

35. Vahala, K. J. Optical microcavities. Nature 424, 839-846 (2003).

36. Cao, H. \& Wiersig, J. Dielectric microcavities: model systems for wave chaos and non-Hermitian physics. Rev. Mod. Phys. 87, 61 (2015).

37. Ward, J. \& Benson, O. WGM microresonators: sensing, lasing and fundamental optics with microspheres. Laser Photonics Rev. 5, 553-570 (2011).

38. Curcio, J. A. \& Petty, C. C. The near infrared absorption spectrum of liquid water. J. Optical Soc. Am. 41, 302-304 (1951).

39. Ward, J. M. et al. Nanoparticle sensing beyond evanescent field interaction with a quasi-droplet microcavity. Optica 5, 674-677 (2018).

40. Graziano, G. On the temperature-induced coil to globule transition of poly-Nisopropylacrylamide in dilute aqueous solutions. Int. J. Biol. Macromol. 27, 89-97 (2000).

41. Chen, Z. M. et al. Packaged microbubble resonator optofluidic flow rate sensor based on Bernoulli effect. Opt. Express 27, 36932-36940 (2019).

42. Lu, Y. J. et al. Origin of hysteresis observed in association and dissociation of polymer chains in water. Phys. Chem. Chem. Phys. 12, 3188-3194 (2010).

43. Sun, B. J. et al. A FTIR and 2D-IR spectroscopic study on the microdynamics phase separation mechanism of the poly( $N$-isopropylacrylamide) aqueous solution. Macromolecules 41, 1512-1520 (2008).

44. Ding, Y. W., Ye, X. D. \& Zhang, G. Z. Microcalorimetric investigation on aggregation and dissolution of poly( $N$-isopropylacrylamide) chains in water. Macromolecules 38, 904-908 (2005).

45. Gomez-Galvan, F. \& Mercado-Uribe, H. The phase transition of poly ( $N$ isopropylacrylamide): the effect of aging. Phase Transit. 87, 336-343 (2014).

46. Grinberg, N. V. et al. Studies of the thermal volume transition of poly $(N$ isopropylacrylamide) hydrogels by high-sensitivity differential scanning microcalorimetry. 1. Dyn. Eff. Macromol. 32, 1471-1475 (1999).

47. Higham, A. K et al. Photo-activated ionic gelation of alginate hydrogel: realtime rheological monitoring of the two-step crosslinking mechanism. Soft Matter 10, 4990-5002 (2014).

48. Wu, C. \& Zhou, S. Q. First observation of the molten globule state of a single homopolymer chain. Phys. Rev. Lett. 77, 3053-3055 (1996). 
49. Rios, C. et al. Integrated all-photonic non-volatile multi-level memory. Nat. Photonics 9, 725-732 (2015).

50. Qu, Y. R. et al. Thermal camouflage based on the phase-changing material GST. Light 7, 26 (2018).

51. Du, K. K. et al. Control over emissivity of zero-static-power thermal emitters based on phase-changing material GST. Light 6, e16194 (2017).

52. Moss, B. et al. Linking in situ charge accumulation to electronic structure in doped $\mathrm{SrTiO}_{3}$ reveals design principles for hydrogen-evolving photocatalysts. Nat. Mater. 20, 511-517 (2021).

53. Yoon, $\mathrm{H}$. et al. Reversible phase modulation and hydrogen storage in multivalent $\mathrm{VO}_{2}$ epitaxial thin films. Nat. Mater. 15, 1113-1119 (2016).
54. Lopez, C. G. \& Richtering, W. Does Flory-Rehner theory quantitatively describe the swelling of thermoresponsive microgels? Soft Matter 13, 8271-8280 (2017).

55. Hirotsu, S. Static and time-dependent properties of polymer gels around the volume phase transition. Phase Transit. 47, 183-240 (1994).

56. Lopez-Leon, T. \& Fernandez-Nieves, A. Macroscopically probing the entropic influence of ions: deswelling neutral microgels with salt. Phys. Rev. E 75, 011801 (2007).

57. Hirotsu, S. et al. Brillouin scattering study of the volume phase transition in poly-N-isopropylacrylamide gels. J. Phys. Soc. Jpn. 64, 2898-2907 (1995). 